\title{
HepaRG Maturation in Silk Fibroin Scaffolds: Toward Developing a 3D In Vitro Liver Model
}

\author{
Alycia Abbott and Jeannine M. Coburn \\ Department of Biomedical Engineering, Worcester Polytechnic Institute, Worcester, MA
}

\section{Total Pages: 6}

\section{List of supporting figures:}

Figure S1. Determination of HepaRG Cell Seeding Amount

Figure S2. H\&E images from Day 14 of functionalized silk scaffold experiments

Figure S3. Day 28 gene expression and albumin secretion by HepaRG cells after 28 days of culture on the functionalized silk scaffolds

Figure S4. Determination of Oleic Acid Concentration and Time of Exposure

\section{List of supporting tables:}

Table S1. List of primers used for qPCR 
A
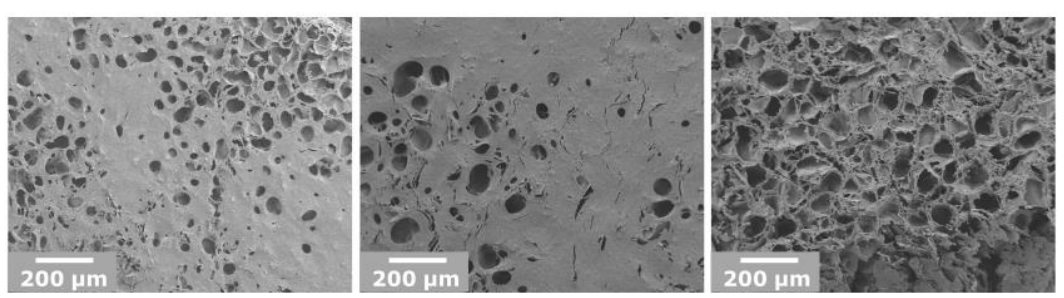

10,000 Cells

20,000 Cells

30,000 Cells
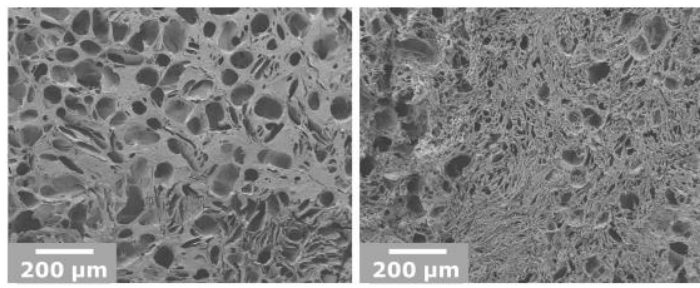

40,000 Cells

50,000 Cells

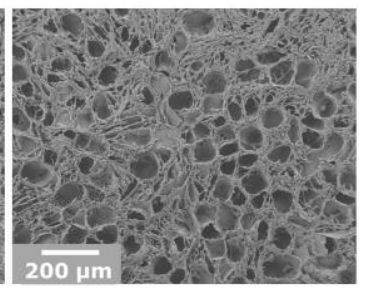

60,000 Cells

B

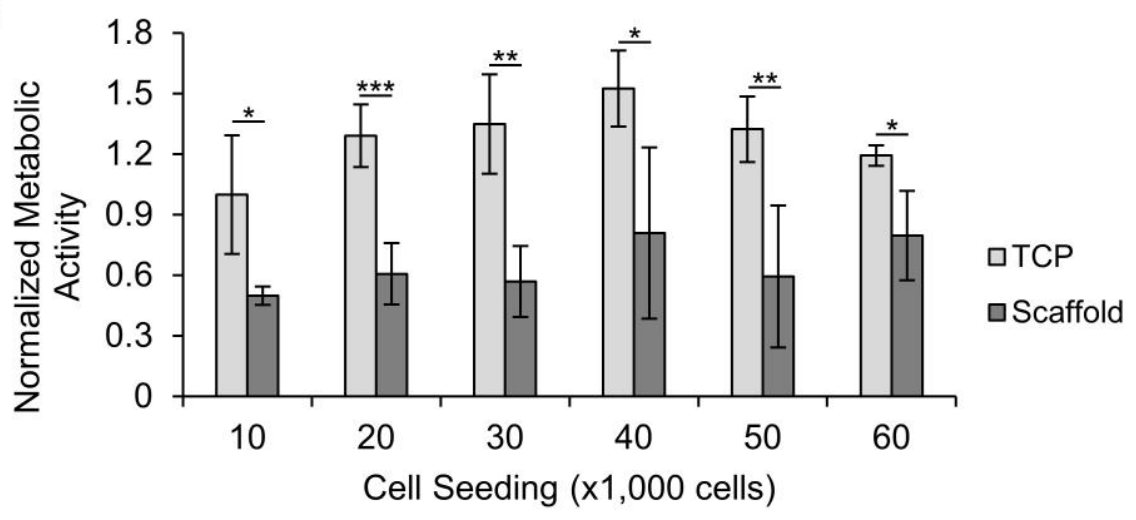

Figure S1. Determination of HepaRG cell seeding number. (A) Day 7 SEM images (100x) from cell seeding density experiments. (B) Normalized fluorescence of resazurin after incubation with HepaRG cells on Day 7. All values normalized to average fluorescence for 10,000 TCP HepaRG cell group and presented as mean \pm SD from four independent samples. Metabolic activity was significantly greater in TCP HepaRG cells than scaffolded HepaRG cells as determined by a Student's $t$ test $\left({ }^{*} p<0.05,{ }^{* *} p<0.01 ;{ }^{* * *} p<0.001\right)$. 


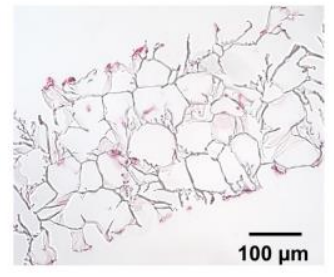

S

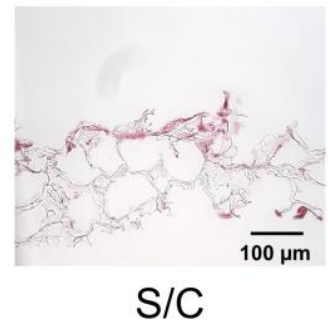

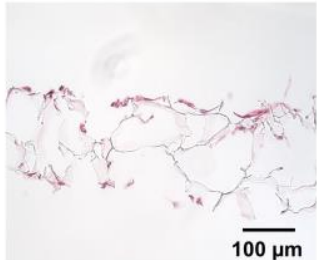

$\mathrm{S}+\mathrm{A}$

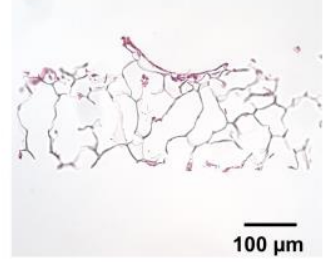

$\mathrm{S} / \mathrm{C}+\mathrm{A}$

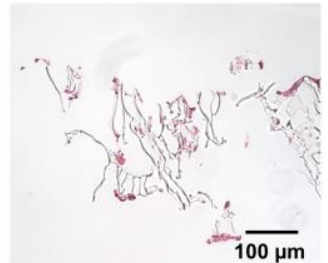

$\mathrm{S}+\mathrm{A}+\mathrm{BRGD}$

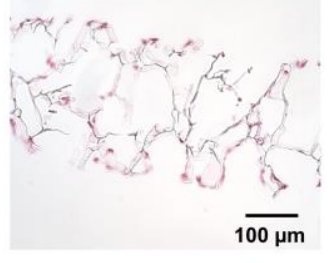

$\mathrm{S} / \mathrm{C}+\mathrm{A}+\mathrm{BRGD}$

Figure S2. H\&E images of Day 14 scaffolded HepaRG cells on different functionalized silk scaffolds demonstrating cell infiltration throughout the scaffolds. Figure Key: $S=$ silk; $S+A$ $=$ silk + avidin; $\mathrm{S}+\mathrm{A}+\mathrm{BRGD}=$ silk + avidin $+\mathrm{B}-\mathrm{RGD} ; \mathrm{S} / \mathrm{C}=$ silk/collagen; $\mathrm{S} / \mathrm{C}+\mathrm{A}=$ silk/collagen + avidin; $\mathrm{S} / \mathrm{C}+\mathrm{A}+\mathrm{BRGD}=$ silk/collagen + avidin + B-RGD. 

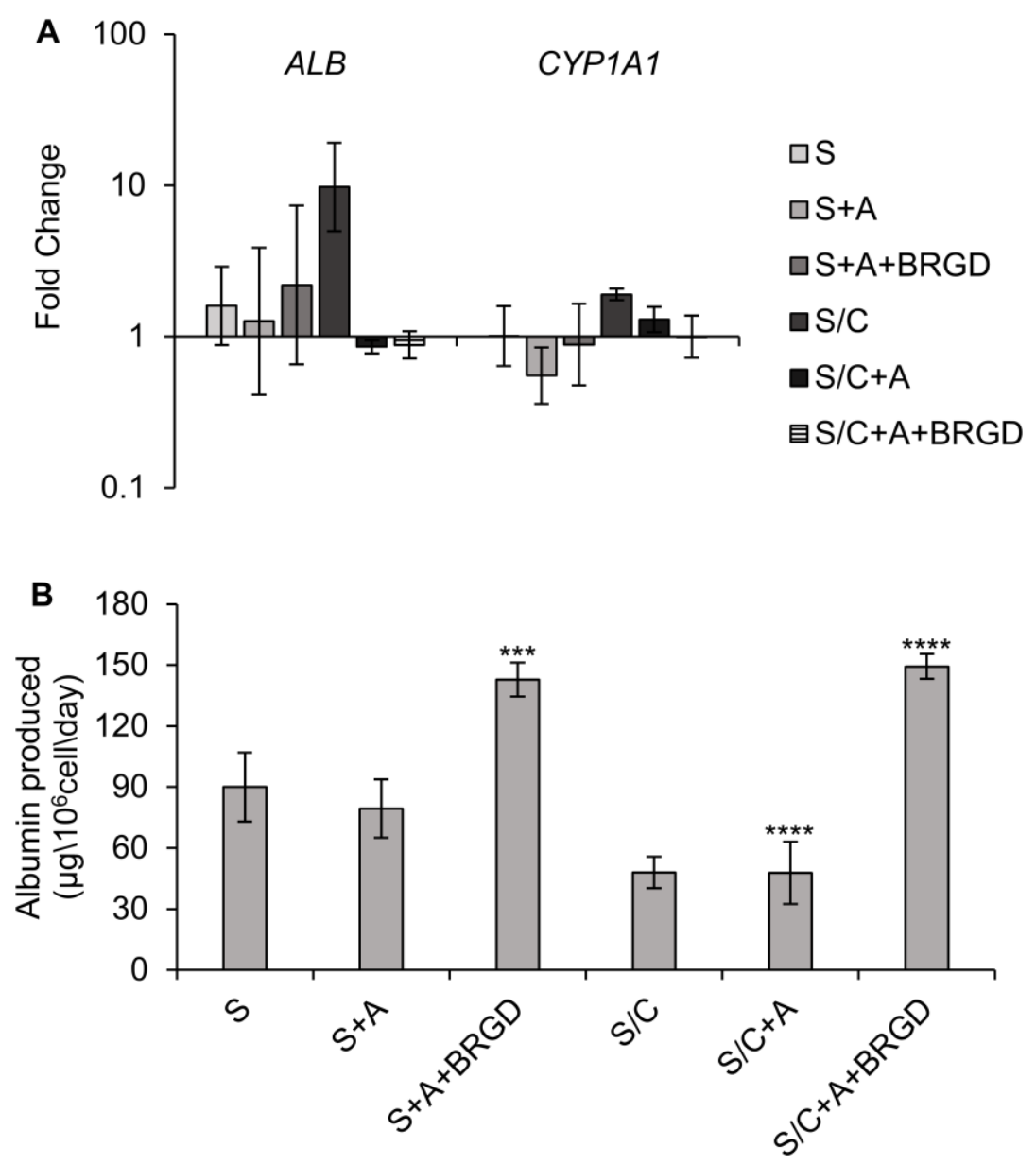

Supplemental Figure 3. Day 28 gene expression and albumin secretion of HepaRG cells in the different silk scaffold functionalization groups. (A) Gene expression data of scaffolded HepaRG cells at Day 28 normalized to scaffolded Day 14 cells. Data are presented as mean $\pm S D$ of three independent experiments. (B) Average albumin secretion over cell culture period. Data are presented as mean $\pm S D$ from three independent samples Asterisks indicate significant difference from $S$ as determined by an ANOVA followed by a Dunnett's test $\left({ }^{* * *} p<0.001,{ }^{* * * *} p<0.0001\right)$. Figure Key: $S=$ silk; $S+A=$ silk + avidin; $S+A+B R G D=$ silk + avidin + B-RGD; $\mathrm{S} / \mathrm{C}=$ silk/collagen; $\mathrm{S} / \mathrm{C}+\mathrm{A}=$ silk/collagen + avidin; $\mathrm{S} / \mathrm{C}+\mathrm{A}+\mathrm{BRGD}=$ silk/collagen + avidin + B-RGD. 

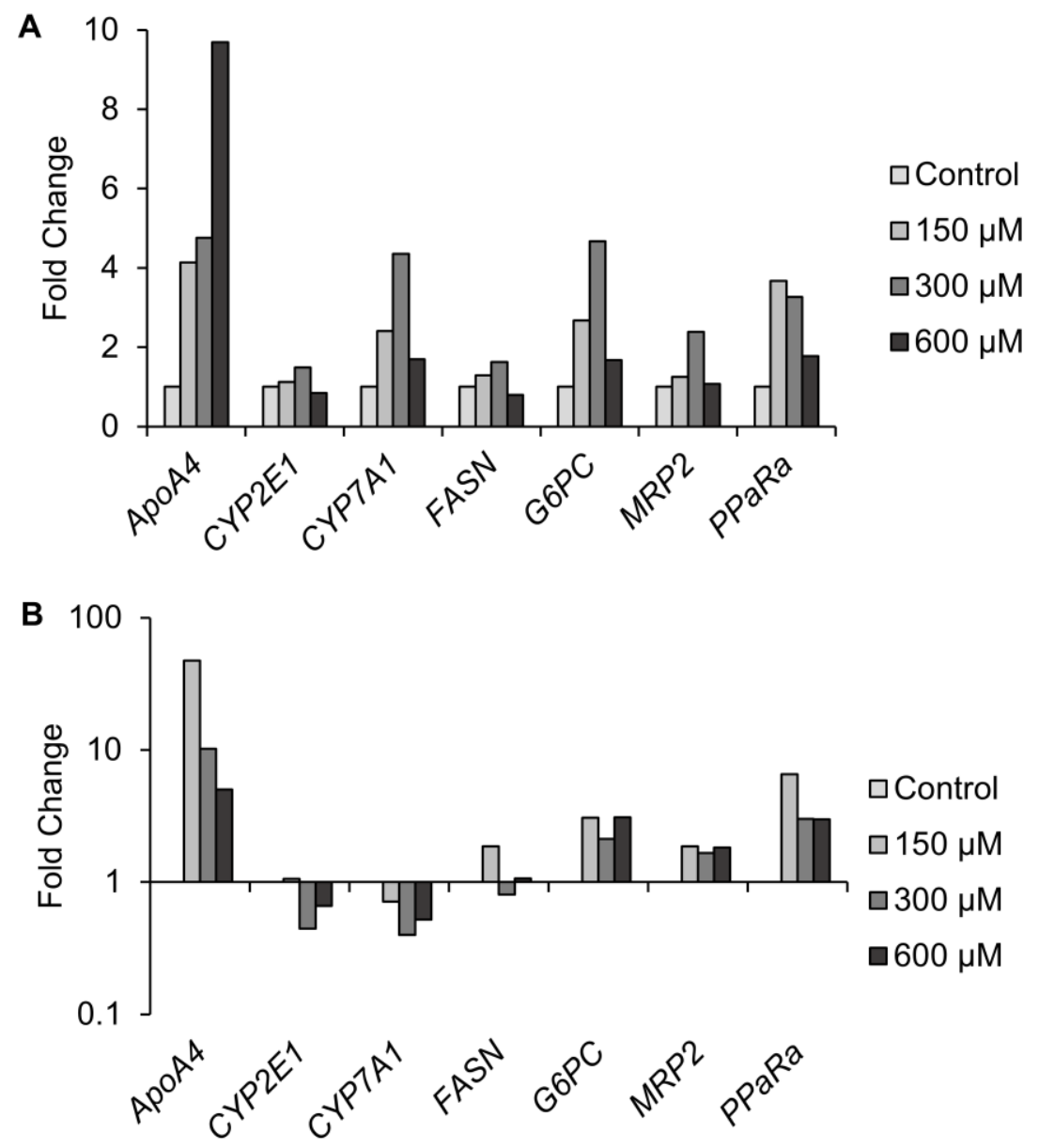

Supplemental Figure 4. Determination of oleic acid concentration and exposure time. Gene expression of TCP HepaRG cells after exposure to 0 (Control), 150, 300, or $600 \mu \mathrm{M}$ oleic acid for (A) 24 or (B) 96 hours. Oleic acid treated groups are normalized to control TCP HepaRG cells. 
Table S1. List of primers used for qPCR

\begin{tabular}{|c|c|c|}
\hline Gene & Accessor Transcript & Sequence \\
\hline$G A P D H$ & NM_001289746.1 & $\begin{array}{l}\text { F - TGC ACC ACC AAC TGC TTA GC } \\
\text { R - GGC ATG GAC TGT GGT CAT GAG }\end{array}$ \\
\hline ACSL4 & NM_004458.3 & $\begin{array}{c}\text { F - TTT TTG CGA GCT TTC CGA GTG } \\
\text { R - TGC CAT AGC GTT TTT AAA TGT CC }\end{array}$ \\
\hline$A L B$ & NM_000477.5 & $\begin{array}{l}\text { F - GCA CAA TGA AGT GGG TAA CC } \\
\text { R - CAG CAG TCA GCC ATT TCA CC }\end{array}$ \\
\hline ApoA4 & NM_000482 & $\begin{array}{c}\mathrm{F} \text { - CAG TGT GGC AAG AAA CTC CT } \\
\mathrm{R} \text { - GTA GTC CCA CAT CAC CGT G }\end{array}$ \\
\hline CK19 & NM_002276.4 & $\begin{array}{l}\text { F - AGC ATG AAA GCT GCC TTG GA } \\
\text { R - CCT GAT TCT GCC GCT CAC TAT C }\end{array}$ \\
\hline CYP1A1 & NM_001319217.1 & $\begin{array}{l}\mathrm{F} \text { - TCC AGA GAC AAC AGG TAA AAC A } \\
\mathrm{R}-\mathrm{AGG} \text { AAG GGC AGA GGA ATG TGA T }\end{array}$ \\
\hline CYP2E1 & NM_000773 & $\begin{array}{l}\text { F - TTG AAG CCT CTC GTT GAC CC } \\
\text { R - GTG GGA TAC AGC CAA ACC CA }\end{array}$ \\
\hline CYPЗА4 & NM_017460.5 & $\begin{array}{l}\text { F - AAA CCG GAG GCC TTT TGG TC } \\
\text { R - TGG TGA AGG TTG GAG ACA GC }\end{array}$ \\
\hline FASN & NM_004104.5 & $\begin{array}{l}\mathrm{F} \text { - GTA CAC ACC CAA GGC CAA GTA } \\
\text { R - GAC GTG GAC GGA TAC TTT CC }\end{array}$ \\
\hline Glut2 & NM_001278658.2 & $\begin{array}{l}\text { F - ATC AAT GCA CCT CAA CAG AAT CA } \\
\text { R - CCC ATC AAG AGA GCT CCA ACT A }\end{array}$ \\
\hline G6PC & NM_000151 & $\begin{array}{c}\text { F - CTT CCG TGC CCC TGA TAA AG } \\
\text { R - GTA GGT CGG CTT TAT CTT TCC C }\end{array}$ \\
\hline HADHA & NM_000182.5 & $\begin{array}{l}\text { F - TTC AGC AAC TTG ACT GGG CA } \\
\text { R - CAG TGA TCT GGA ATC ACC GCT }\end{array}$ \\
\hline$H N F \alpha$ & NM_001258355.1 & $\begin{array}{l}\text { F - GCT CGG AGC CAC CAA GAG AT } \\
\text { R - CGT ATG GAC ACC CGG CTC AT }\end{array}$ \\
\hline IRS1 & NM_005544.3 & $\begin{array}{l}F-A C T \text { GGA CAT CAC AGC AGA ATG A } \\
R \text { - ACT GAA ATG GAT GCA TCG TAC C }\end{array}$ \\
\hline MRP2 & NM_000392 & $\begin{array}{l}\text { F - TGC TTC CTG GGG ATA ATC AG } \\
\text { R - CAC GGA TAA CTG GCA AAC CT }\end{array}$ \\
\hline MDR3 & NM_018849 & $\begin{array}{l}\text { F - CTG GAG GTG AAG AAA GGC CA } \\
\text { R - CCA AAG TCC ACA AAC ACT GTC C }\end{array}$ \\
\hline MDR4 & NM_005845.5 & $\begin{array}{l}\text { F - CCG TGT ACC AGG AGG TGA AG } \\
R \text { - ACA AGG GAT TGA GCC ACC AG }\end{array}$ \\
\hline SCARB1 & NM_001082959.2 & $\begin{array}{l}F-G A A \text { GGC ATC CCC ACC TAT CG } \\
R \text { - AGA AAC AAG GGG GCA CTG AA }\end{array}$ \\
\hline SLC10A1 & NM_003049.4 & $\begin{array}{l}\text { F - TGC GCT ATG TCA TCA AGG GAG } \\
\text { R - TGG CAG AGA GAA CTG TGA CG }\end{array}$ \\
\hline PCK2 & NM_001308054.2 & $\begin{array}{l}\text { F - ACC TCC CTC GGA CCT CTA AC } \\
\text { R - TGC CAG TTA AGC CCT TGT CC }\end{array}$ \\
\hline$P P a R \alpha$ & NM_001362873 & $\begin{array}{l}F-A C T \text { CTT GCG AGG GAT CTC GG } \\
R \text { - ACA ACC CGA AGA CCC AGA GT }\end{array}$ \\
\hline
\end{tabular}

